Bull. Mater. Sci., Vol. 6, No. 3, July 1984, pp. 453-458. (C) Printed in India.

\title{
High resolution electron microscopy of superconductors
}

\author{
C S PANDE \\ Naval Research Laboratory, Washington D.C. 20375, USA
}

\begin{abstract}
Until recently, in the field of superconductivity, electron microscopy has played only a minor role in studies of structure and properties of existing superconductors (except $\mathrm{Nb}$ and its alloys) and in the development of new and improved superconductors. This situation has changed in recent years. Electron microscopy is now being extensively used to study the growth, microstructure, and kinetics of many superconductors, especially of A15 type. Electron microscopy has also contributed to the understanding of the nature of radiation damage in these materials. These contributions will be reviewed with selected examples.
\end{abstract}

Keywords. Electron microscopy; super conducting materials; radiation damage; defect structure.

\section{Introduction}

Electron microscopy has now developed as one of the most powerful techniques available to metallurgists and solid state physicists. It is capable of very high resolution $((0.2-0.3 \mathrm{~nm})$ lattice resolution, or $\sim 0.5 \mathrm{~nm}$ point to point resolution is routine in modern conventional electron microscopes). Electron microscopes with a lattice resolution as fine as $0.06 \mathrm{~nm}$ is now available commercially. Crystal lattice of virtually most of the materials can now be resolved at least in principle by these instruments. However, an alternative mode of operation, i.e. the diffraction contrast is the most popular technique in use. This images the lattice defects in the lattice with a poorer resolution $(\sim 10 \mathrm{~nm})$. However, the use of the weak beam technique has pushed this limit to $\sim 2 \mathrm{~nm}$. In addition, most electron microscopes display electron diffraction information. In recent years an attachment to the conventional microscope has been developed that can be used to convert it to a scanning electron microscope with a electron beam as fine as $1.5 \mathrm{~nm}$. (This is in addition to dedicated scanning transmission electron microscopes (STEM) which are now commercially available with electron beam capabilities as fine as atomic dimensions. With the use of such a fine beam, individual atoms of some heavy elements have been photographed for the first time.) The availability of the fine electron beam in these instruments has opened up new possibilities, such as the use of energy dispersive $x$-ray spectroscopy and electron energy loss spectroscopy to obtain chemical analysis of regions as small as $5 \mathrm{~nm}$. All these techniques can often be used for the same region of the specimen leading to a complete characterization of the given material (Hren et al 1979).

In spite of the availability of all these techniques, electron microscopy has, until recently, played only a minor role in studies of the structure and properties of existing superconductors and in the development $o_{2}$ ' new and improved superconducting materials. Until 1974, most of the published work in this field related to the studies in $\mathrm{Nb}$ and its alloy, including the commercial material $\mathrm{Nb}-\mathrm{Ti}$. Very little attention has been paid to $\mathrm{A} 15$ compounds such as $\mathrm{Nb}_{3} \mathrm{Sn}$ which are now beginning to replace $\mathrm{Nb}-\mathrm{Ti}$ 
in technological applications. One possible reason for this was the difficulty of preparing thin film specimens from bulk for many superconducting materials as they are very brittle. Section 2 describes in some detail specimen preparation techniques followed by the applications of high resolution electron microscopy to high $T_{c}$ (superconducting transition temperature) materials especially A15 materials. Some applications of high resolution electron microscopy to specimens cooled below $T_{c}$ in the electron microscopes will also be mentioned.

\section{Specimen preparation}

For conventional $(100 \mathrm{kV})$ transmission electron microscopy the specimens have to be less than $200 \mathrm{~nm}$. It is, therefore, often necessary to obtain foils of such thickness from bulk. (Superconductors have also been prepared by vacuum evaporation or sputtering where in many cases one can deposit the right thickness directly avoiding any need for thinning.) For elemental superconductors such as $\mathrm{Nb}$ and its alloys, the conventional techniques such as electrolytic thinning, or chemical thinning, gives excellant specimens. These techniques are, however, not suitable for most of the high $T_{c}$ superconductors. For these materials the most suitable technique is ion milling. Ion milling is essentially a way of thinning specimens by bombardment with inert heavy ions such as Argon ions. Care, however, is required in minimizing radiation damage and surface roughness. Typically the ion damage extends to about $5 \mathrm{~mm}$ below the surface of the specimen. (For full details of the technique see the review by Pande 1979a).

\section{Application of high resolution transmission electron microscopy}

\subsection{Niobium and its alloys}

High resolution transmission electron microscopy of ductile type II superconductors such as $\mathrm{Nb}, \mathrm{NbBi}$ and $\mathrm{Nb}-\mathrm{Zr}$ have been mostly directed towards understanding the flux pinning behaviour of these materials. Flux pinning and hence, critical current $J_{c}$, is very dependent on the interaction between the Abrikosov vortices (present in these materials in superconducting state) and the lattice defects, such as dislocations and precipitates. TEM has been used to characterize the defect structure. For example, Narlikar and Dew-Hughes (1966) showed that when the dislocations are uniformly distributed in $\mathrm{Nb}$ and its alloys (as observed by TEM) they have only a weak effect on $J_{c}$ due probably to weak pinning of flux lines. Stronger pinning was observed when the dislocations were clustered into walls enclosing dislocation free regions (such as obtained after cold working). This result was also true for drawn superconducting filaments. The size of these cells (enclosed by the dislocation walls) was also significant. Neal et al (1971) found that in $\mathrm{Nb}$-Ti highest $J_{c}$ can also be increased by second phase precipitation. TEM showed that $J_{c}$ is highest when the precipitate dispersion was finest. Further, it was found by Milne and Finlayson (1972) that the $J_{c}$ value in $\mathrm{Nb}-\mathrm{Zr}$ alloys depended on the volume fraction of the precipitates. Hillmann and Hauck (1972) using TEM discovered an interesting effect, the so-called "matching effect." $J_{c}$ was highest when there was a matching between the flux line lattice spacing and the distance between the pinning sites. 


\subsection{A15 superconductors}

3.2a Martensitic phase transformations: One of the earliest applications of TEM in the study of A15 compounds was the direct observation of martensitic phase transformation in $\mathrm{V}_{3} \mathrm{Si}$. The transformation was observed as a thinned $\mathrm{V}_{3} \mathrm{Si}$ specimen cooled below $20 \mathrm{~K}$ in a liquid helium stage inside an electron microscope. Twin-related lamellae with thicknesses as small as $10 \mathrm{~nm}$ were observed (Goringe and Valdre 1966). A similar though not as extensive observation was made in $\mathrm{V}_{3} \mathrm{Ga}$ (Nembach and Tachikawa 1970).

$3.2 b$ Flux pinning by grain boundaries: The high $J_{c}$ of $\mathrm{A} 15$ compounds has been ascribed to fine grain size in these materials. Figure 1 shows fine grains in bronze processed $\mathrm{Nb}_{3} \mathrm{Sn}$. The first experimental correlation between grain size and $J_{c}$ on the basis of TEM observation, was given by Nembach and Tachikawa (1970) in $\mathrm{V}_{3} \mathrm{Ga}$, and by Scanlan et al (1975) in $\mathrm{Nb}_{3} \mathrm{Sn}$. Extensive TEM on bronze processed $\mathrm{Nb}_{3} \mathrm{Sn}$ showed that the grain size in this material could be as small as $40 \mathrm{~nm}$ (Pande unpublished). There were no other major pinning centres such as dislocations and precipitates, even at the grain boundaries. Figure 1 shows transmission electron micrograph showing typical grains in bronze processed $\mathrm{Nb}_{3} \mathrm{Sn}$. It is, therefore, concluded that the pinning in this material is mostly by the grain boundaries. A theory based on this idea has been proposed by Pande and Suenaga (1976).

3.2c Stacking faults: Essmann and Zerweck (1973) have predicted stacking faults on $\{111\}$ planes in A15 structure. In actual practice stacking faults are only observed if a small amount of carbon is added to A15 material (Uzel and Diepers 1973). This presumably implies that interstitial impurities are needed to form these faults.

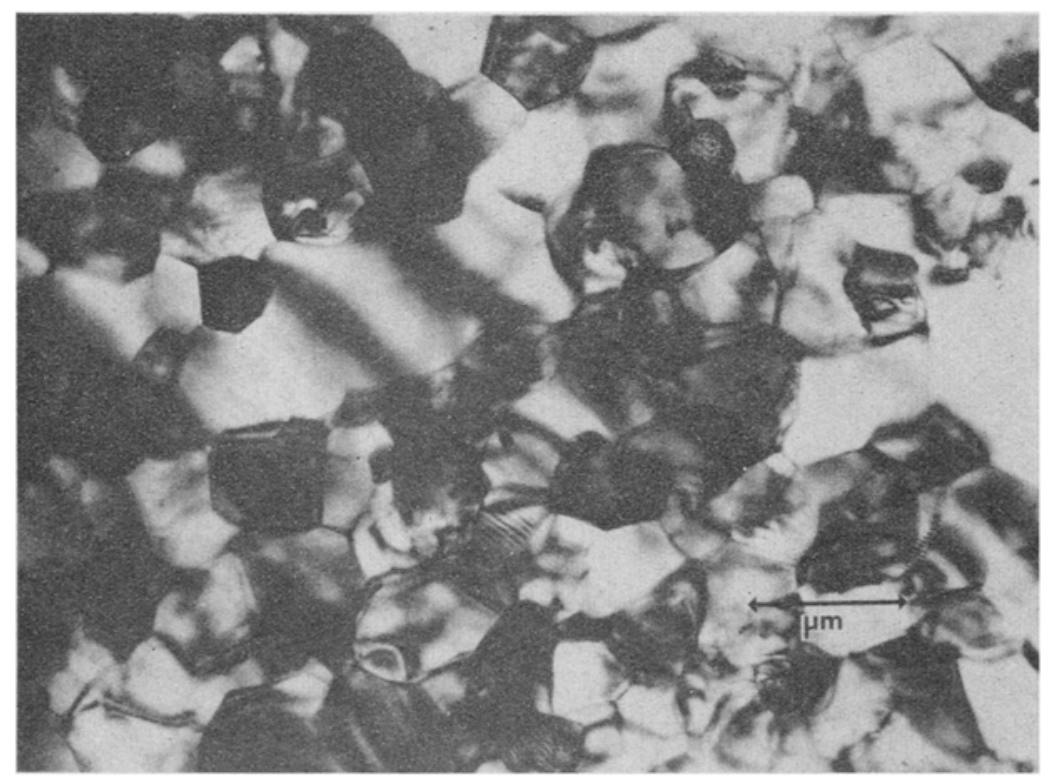

Figure 1. Transmission electron micrograph of bronze processed $\mathrm{Nb}_{3} \mathrm{Sn}$, showing that the most prominent defects are just grain boundaries as pinning centers. 
3.2d Radiation damage: Radiation damage in high $T_{c}$ superconducting materials is of technological concern because of the possible use of superconductors as magnets in fusion reactors. The nature of the damage in A15 compounds due to high energy nuclear radiation has been a subject of much controversy. The defects introduced by these radiations affect both $J_{c}$ and $T_{c}$ in these materials. A systematic high resolution electron microscopy of neutron irradiation of $\mathrm{Nb}_{3} \mathrm{Sn}$ has now clearly revealed (Pande $1979 \mathrm{~b})$ the nature of this defect. Figure 2 shows a transmission electron micrograph imaged in superlattice reflection. This reflection "maps" the region of low disorder. The micrograph shows regions of "disorder" of size $\sim 3.5 \mathrm{~nm}$ in a more or less ordered matrix. These regions were also regions of strain as proved by imaging these defects in fundamental reflections. The defects were coherent with the matrix. A detailed theory of $T_{c}$ degradation based on these observations is given by Pande (1977).

It is not out of place to mention here that early electron microscopes (Ca 1965) had already given some indications of the existence of these disordered regions, although their exact nature could not be ascertained (Cody, Private Communication). These observations were, however, ignored leading to much confusion and controversy.

3.2e Grain growth in multifilamentary superconductors: Multifilamentary superconductors have been increasingly used in recent years. The nature of grain growth in these superconductors is important since it determines the $J_{c}$ value (figure 3 ). These specimens are difficult to thin, except by ion milling. Pande (unpublished) has made an extensive study of the growth of grains as a function of annealing time and temperature.

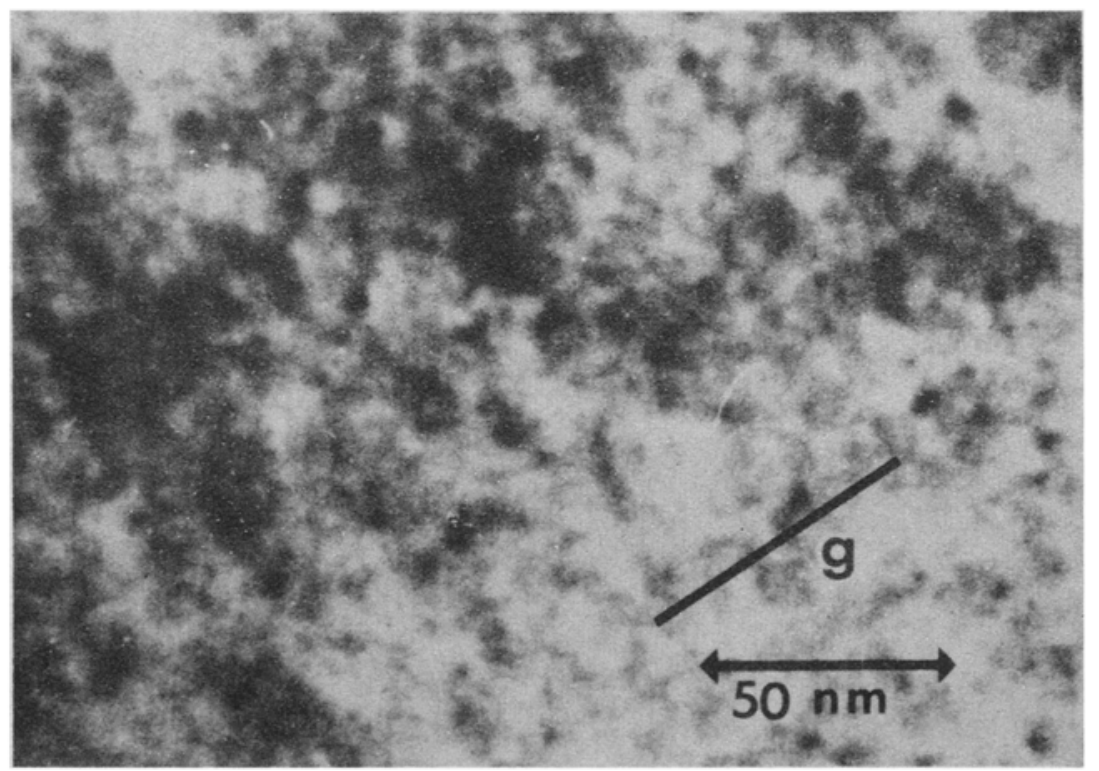

Figure 2. Electron micrograph in superlattice reflection $g=011$ showing small disordered regions of size $3.5 \mathrm{~nm}$ in fast neutron irradiated $\mathrm{Nb}_{3} \mathrm{Sn}$. 


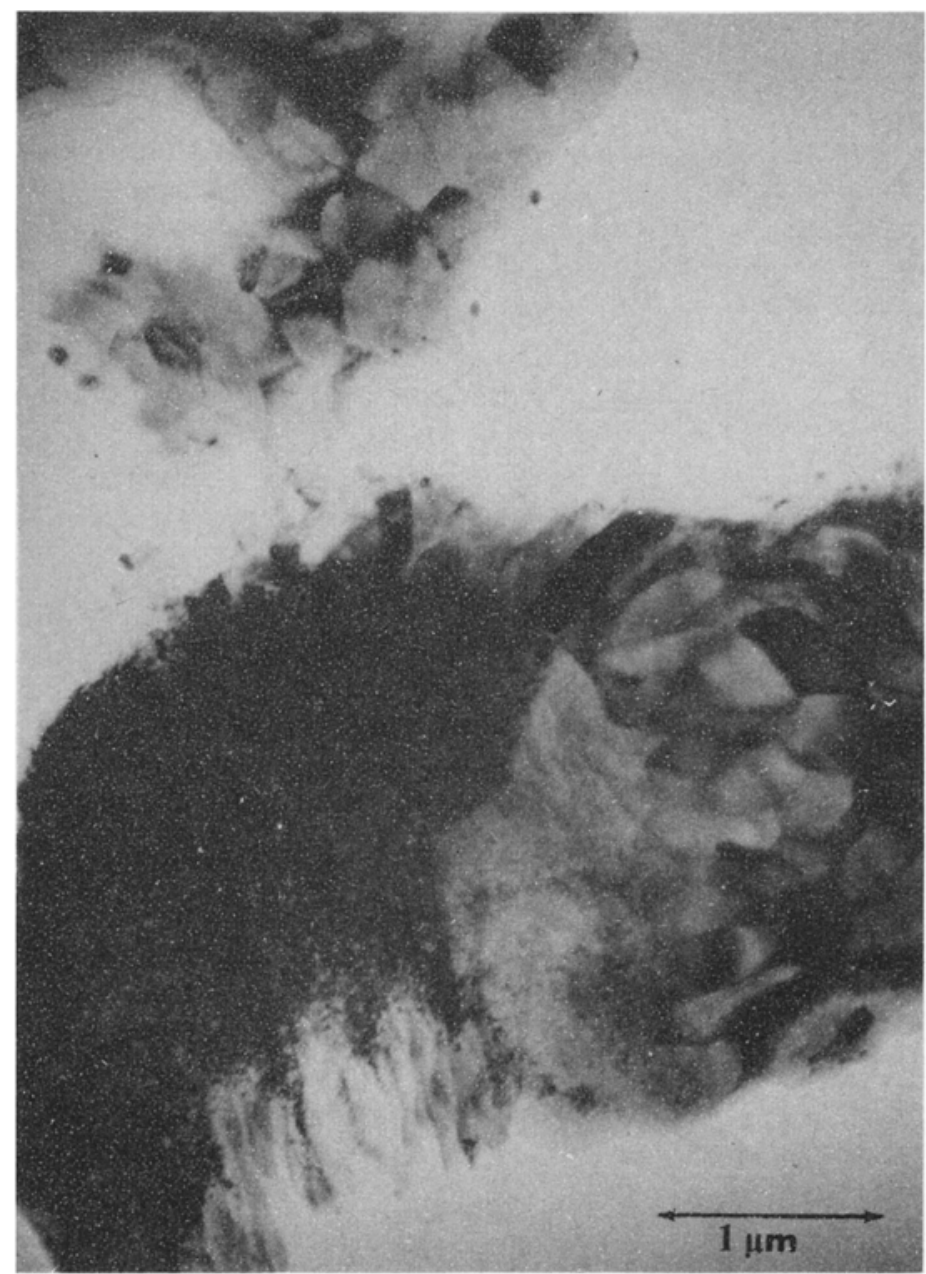

Figure 3. Electron micrograph of filamentary superconductor showing $\mathrm{Nb}_{-} \mathrm{Nb}_{3} \mathrm{Sn}$ and $\mathrm{Sn}-$ $\mathrm{Nb}_{3} \mathrm{Sn}$ interface as well as individual grains. The grains near the interface are elongated.

$3.2 f$ Dislocations in A15 compounds: The dislocations in A15 compounds do not play any major role. However, the nature of dislocations in these materials is of theoretical interest. TEM observations indicate that Burgers vector of many of the dislocations are of the type $b=[100]$.

$3.2 \mathrm{~g}$ Electron diffraction studies: The electron diffraction studies of A15 have contributed a great deal of information regarding imperfections, etc. Schmidt et al (1976) have postulated the existence of charge density waves in these materials. However, a detailed study by Pande (unpublished) showed no evidence of such waves. However, some of the electron diffraction patterns are similar to that obtained by them (see figure 4).

3.2h Direct resolution of flux lines without decoration: Finally, we would like to mention another use of TEM, which has as yet not been successful, i.e. the direct 


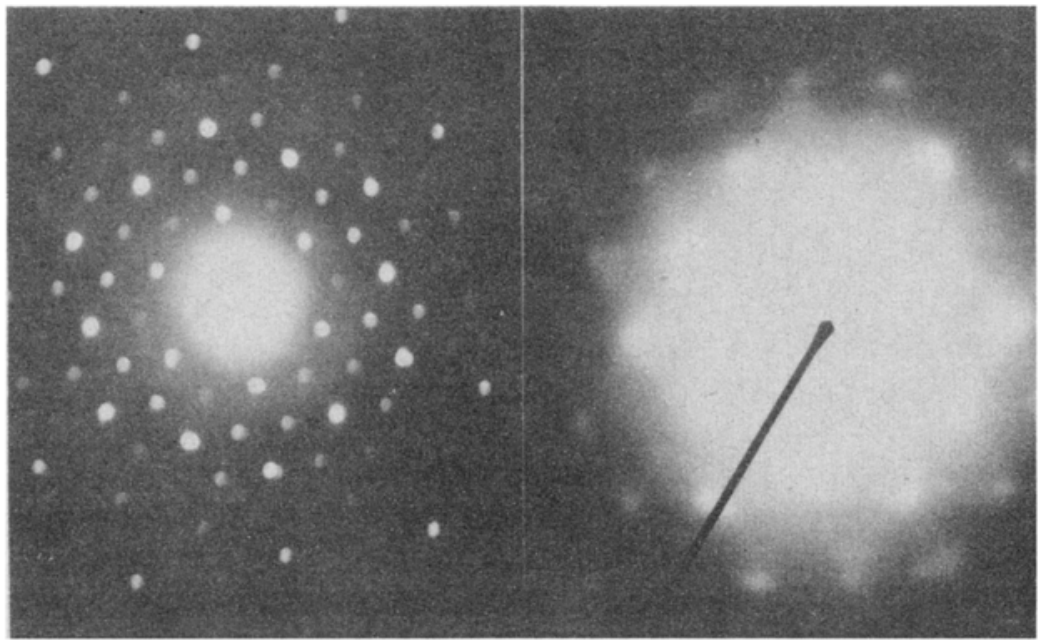

Figure 4. Electron diffraction from a well ordered (left) and heavily disordered (during growth) (right) specimens.

observation of flux line lattice, although thin film techniques have been combined with "decoration" techniques to give good indications of deflect flux line interactions (Herring 1974).

It is hoped that the examples presented here have demonstrated the usefulness of electron microscopy in a study of A15 superconductors. Much valuable information has already been obtained. It is expected that high resolution electron microscopy will be increasingly used in characterizing and developing technologically important superconductors.

\section{References}

Cody G D Private communication

Essmann U and Zerweek G 1973 Phys. Status Solidi B57 611

Goringe M J and Valdre U 1966 Proc. R. Soc. (London) A295 869

Herring C 1974 Phys. Lett. A47 105

Hillmann H and Hauck D 1972 Appl. Superconductivity Conf., (New York: Institute of Electrical and Electronics Engineers) 429

Hren J J, Goldstein J I and Joy D C 1979 Introduction to analytical electron microscopy (New York: Plenum Press)

Milne I and Finlayson T R 1972 Appl. Superconductivity Conf. (New York: Institute of Electrical and Electronics Engineers) 425

Narlikar A V and Dew-Huger D 1966 J. Mater. Sci. 1317

Neal D F, Barber A C, Woolcock A and Gidley J A F 1971 Acta Metallur. 19143

Nembach E and Tachikawa K 1970 Philos. Mag. 21869

Pande C S 1977 Solid State Commun. 24241

Pande C S 1979a Superconductivity and electron microscopy in treatise on material science and technology' 14 171

Pande C S 1979b Phys. Status. Solidi A52 687

Pande C S (Unpublished work)

Pande C S and Suenaga M 1976 Appl. Phy's. Lett, 29443

Scanian R M, Fietz W A and Kich E F 1975 J. Appl. Phys. 462244

Schmidt P H, Spencer E G, Joy D C and Rowell J M 1976 2nd Conf. Superconductirity ind-and f-band Met. (ed) D H Douglas (New York: Plenum Press) 431

Uzel Y and Diepers H 1973 Z. Phy's. 258126 